\title{
Qualificação dos serviços farmacêuticos no Brasil: aspectos inconclusos da agenda do Sistema Único de Saúde
}

\author{
Fabiola Sulpino Vieira ${ }^{1}$
}

Como citar Vieira FS. Qualificação dos serviços farmacêuticos no Brasil: aspectos inconclusos da agenda do Sistema Único de Saúde. Rev Panam Salud Publica. 2008:24(2):91-100.

RESUMO Objetivos. Apontar aspectos inconclusos da agenda do Sistema Único de Saúde (SUS) para a qualificação dos serviços farmacêuticos no Brasil.

Métodos. Os dados foram coletados a partir dos relatórios de fiscalização de municípios elaborados pela Controladoria Geral da União (CGU). Utilizaram-se os relatórios completos de fiscalizações realizadas entre agosto de 2004 e julho de 2006, disponíveis na Internet. Todos os relatórios publicados foram analisados. Os problemas identificados foram organizados em 15 categorias. Resultados. Foram realizadas 660 fiscalizações e analisados 659 relatórios (um não estava disponível). Em 62 municípios, os recursos da assistência farmacêutica não foram auditados, $o$ que resultou em uma amostra final de 597 municípios (10,7\% dos municípios brasileiros). Desses, 90,3\% apresentaram problemas na gestão de recursos ou serviços. Em $71 \%$ dos municípios foi constatada a falta de controle de estoque ou sua deficiência e em $39 \%$ condições inadequadas de armazenamento. Verificou-se falta de medicamentos em $24 \%$ dos municípios.

Conclusões. Os propósitos da Política Nacional de Medicamentos e da Política Nacional de Assistência Farmacêutica estão distantes da concretização. O tema deve ser novamente incluído na agenda dos governos nas três esferas, pois não é possível falar em efetividade da atenção à saúde sem que a assistência farmacêutica cumpra o seu papel com qualidade.

Palavras-chave Serviços de assistência farmacêutica, avaliação de processos, Política Nacional de Assistência Farmacêutica, Política Nacional de Medicamentos, Sistema Único de Saúde, Brasil.

A República Federativa do Brasil está organizada político-administrativamente em União, Distrito Federal, 26 estados e 5564 municípios, que pela Constituição de 1988 são todos entes federados autônomos. Entre-

\footnotetext{
1 Ministério da Saúde, Secretaria Executiva, Área de Economia da Saúde e Desenvolvimento. Correspondência: Esplanada dos Ministérios, Bloco G, Ala A, sala 229A, CEP 70058-900, Brasília, DF, Brasil. Fone: + 55-61-3340-4333; e-mail: fabiolasulpino@ uol.com.br
}

tanto, conforme a mesma Constituição, todos esses entes autônomos constituem, no caso da saúde pública, o Sistema Único de Saúde (SUS). O SUS foi criado para executar ações de promoção, prevenção e recuperação da saúde, obedecendo aos princípios de universalidade, igualdade e integralidade do acesso às ações e serviços (1).

A gestão do sistema é realizada pelas três esferas de governo, municipal, esta- dual e federal, com compartilhamento das responsabilidades e do financiamento. Os serviços de saúde são administrados principalmente pelos municípios, que formam, com os serviços sob gestão dos estados e da União, uma rede regionalizada e hierarquizada.

Em relação à assistência farmacêutica, observa-se uma lacuna de 10 anos entre o estabelecimento do SUS e das iniciativas para efetivação dessa assistência como área de atuação do sis- 
tema; o reconhecimento se deu com a elaboração da diretriz constitucional de integralidade das ações e serviços de saúde (1), enquanto as iniciativas de efetivação resultaram da formulação e da implementação de uma política em âmbito federal para sua organização. Ao longo desses 10 anos, pode-se considerar que as demandas para a estruturação da assistência farmacêutica se mantiveram reprimidas. $\mathrm{Na}$ visão de Rua (2), o estado de demandas reprimidas é aquele no qual as demandas para resolução de uma situação-problema existem como um "estado de coisas", ou seja, a situação é desagradável e pode permanecer assim por muito tempo. Nesse estado, embora haja grupos insatisfeitos, não há mobilização das autoridades governamentais para uma ação política. Ainda segundo essa autora, quando uma situação se transforma em problema político, deixa de ser um estado de coisas e passa a fazer parte da agenda do governo (2).

A inclusão da assistência farmacêutica na agenda do Governo Federal no Brasil se deu apenas a partir de 1998 e resultou na publicação da Política Nacional de Medicamentos (PNM) (3). Foram estabelecidas as seguintes diretrizes: adoção de uma lista de medicamentos essenciais, regulamentação sanitária de medicamentos, reorientação da assistência farmacêutica, promoção do uso racional de medicamentos, desenvolvimento científico e tecnológico, promoção da produção, garantia da segurança, eficácia e qualidade dos medicamentos e desenvolvimento e capacitação de recursos humanos.

A partir de então, o Ministério da Saúde intensificou a estruturação de programas de assistência farmacêutica, com o propósito de melhorar o acesso da população aos medicamentos, seja por meio de aquisição direta e envio desses medicamentos aos municípios, seja pelo repasse de recursos financeiros aos municípios para aquisição de medicamentos.

Em 1999, por exemplo, foi instituído o "Incentivo Financeiro a Municípios Habilitados à Parte Variável do Piso de Atenção Básica (PAB) para a Assistência Farmacêutica Básica". Tratase de uma ação prevista no orçamento do Ministério da Saúde (União) para financiar a aquisição, pelos municípios, de uma lista de medicamentos selecionados para uso na atenção primária à saúde. A responsabilidade do financiamento é compartilhada pelas três esferas de governo (4).

É notório que, no SUS, a estruturação da assistência farmacêutica começou de forma tardia e com descompasso entre os seus componentes técnico (seleção, prescrição, dispensação e uso) e logístico (programação, aquisição, armazenamento e distribuição). Se, de um lado, os programas para oferta de medicamentos começaram a se organizar efetivamente após 10 anos da criação do SUS, por outro, os esforços para reorientação da assistência farmacêutica são ainda mais recentes.

Essa reorientação diz respeito ao deslocamento do foco da assistência farmacêutica estritamente do componente logístico para incluir a melhora da gestão e a qualidade dos serviços e, com isso, a promoção do acesso dos cidadãos aos medicamentos essenciais, com uso racional, tanto do ponto de vista terapêutico quanto do ponto de vista dos recursos públicos. Ou seja: tirar a ênfase do produto para enfocar o usuário dos serviços, equilibrando os dois componentes em uma atuação sistêmica (5). Nesse sentido, em 2001, o Ministério da Saúde publicou materiais técnicos com o objetivo de orientar a organização dos serviços farmacêuticos, por meio do estabelecimento de requisitos técnicos (6).

Posteriormente, na mesma linha da PNM, em 2004 o Conselho Nacional de Saúde publicou o documento da Política Nacional de Assistência Farmacêutica (PNAF). Na PNAF ficam explícitas as necessidades de qualificação dos serviços de assistência farmacêutica e de promoção do uso racional de medicamentos (7). A ratificação da PNAF pelo Ministério da Saúde recolocou a assistência farmacêutica na agenda do Governo Federal. O desdobramento disso se deu em 2006, quando o Ministério da Saúde promoveu 54 Seminários de Apoio ao Planejamento da Assistência Farmacêutica em todo o país, com a participação de estados e municípios, para discutir o planejamento da assistência farmacêutica, e publicou materiais técnicos para instruir a sua organização (inclusive com reedição daquele publicado em 2001) (8-10).

Entretanto, apesar das iniciativas de qualificação, muitos problemas ainda comprometem a qualidade dos serviços farmacêuticos no SUS. Um estudo realizado pelo Ministério da Saúde em parceria com a Organização Pan-Americana da Saúde (OPAS) mostrou que ainda é preciso investir em melhorias (11). Segundo esse estudo, a disponibilidade em estoque dos medicamentos principais foi de $73 \%$ nas unidades de saúde, $76 \%$ nas centrais de abastecimento farmacêutico municipais (CAF-M) e 77\% nas estaduais (CAF-E). Esse resultado mostrou que mesmo os medicamentos considerados imprescindíveis não estavam disponíveis nos serviços. Outro dado importante obtido diz respeito à porcentagem de medicamentos prescritos, dispensados ou administrados, que foi de $66 \%$ nas unidades de saúde, indicando duas situações: ou os medicamentos prescritos não foram dispensados ou administrados porque não estavam disponíveis, ou os prescritores não aderiram às relações de medicamentos selecionados para os serviços de saúde.

Ainda segundo o estudo, o tempo de desabastecimento dos medicamentos principais, medido por meio de fichas de controle de estoque, foi em média de 84 dias nas unidades de saúde, 74 dias nas CAF-M e 128 dias nas CAF-E. No tocante à qualidade dos serviços, em uma escala de 0 a 100 pontos, metade das unidades de saúde alcançaram entre 40 e 69 pontos quanto às boas práticas de estocagem nas centrais de abastecimento farmacêutico, o que evidenciou que os medicamentos poderiam estar sujeitos a condições não adequadas em alguns aspectos nesses serviços. A existência de registros de estoque foi de $32 \%$ nas unidades de saúde, $32 \%$ nas CAF-M e 61\% nas CAF-E, revelando a precariedade do controle de estoque nesses serviços (11).

Corroborando esses achados, o Banco Mundial realizou um estudo 
sobre a governança no SUS e afirmou, em seu relatório publicado em 2007, que o gerenciamento da logística de medicamentos absorve cerca de $20 \%$ dos recursos financeiros da saúde, podendo ser a causa principal de ineficiência e perda (12). O relatório também aponta que há deficiência na qualidade dos serviços, que é atribuída a problemas de gestão e à ineficiência no uso dos recursos.

Dessa forma, considerando que já houve esforço para identificar os problemas de qualidade dos serviços farmacêuticos do SUS, o objetivo deste trabalho foi apontar os aspectos inconclusos da agenda do SUS para a qualificação dos serviços farmacêuticos no Brasil.

\section{MATERIAIS E MÉTODOS}

Os dados sobre os serviços farmacêuticos no SUS foram coletados a partir dos relatórios de fiscalização de municípios, que são os entes federados responsáveis pela execução da maior parcela desses serviços no sistema. Os relatórios de fiscalização foram produzidos pela Controladoria Geral da União (CGU), responsável pelas atividades de controle interno, auditoria pública, correição, prevenção e combate à corrupção.

Para a realização das auditorias e definição dos municípios a serem fiscalizados, a CGU adotou um sistema de sorteio público que utiliza o mesmo modelo da loteria federal. O procedimento é aberto ao público. Antes de cada sorteio, o órgão publica uma norma para definir as regras de cada um deles.

Após o sorteio, os municípios são fiscalizados por servidores da CGU, os quais utilizam parâmetros definidos pelo órgão para a verificação de documentos e averiguação das condições dos serviços oferecidos à população. No caso de identificação de irregularidades, as constatações são discutidas com o gestor responsável, para que este produza contraprovas e apresente justificativas para as situações verificadas. Os relatórios finais das auditorias passaram a ser publicados na íntegra no site eletrônico da CGU (http:// www.cgu.gov.br) a partir do $12^{\circ}$ sorteio. Dessa forma, esta pesquisa utiliza dados das auditorias referentes ao intervalo do $12^{\circ}$ ao $22^{\circ}$ sorteio. A tabela 1 apresenta os critérios utilizados em cada um dos sorteios.

Foram lidos e analisados todos os relatos publicados no período de fevereiro de 2005 a julho de 2007, referentes às fiscalizações feitas entre agosto de 2004 e julho de 2006. Em cada relatório, verificou-se a realização de auditoria da gestão dos recursos da ação Incentivo Financeiro a Municípios Habilitados à parte Variável do Piso de Atenção Básica (PAB) para a Assistência Farmacêutica Básica do Programa Assistência Farmacêutica e Insumos Estratégicos (4) e, conseqüentemente, dos serviços farmacêuticos.

A partir da leitura dos relatos das constatações observadas na execução do programa no município, foram criadas 15 categorias para os problemas identificados, a fim de possibilitar o cálculo de suas freqüências. As categorias criadas foram:

1. secretaria estadual de saúde (SES) não fez contrapartida: para casos em que a SES deixou de alocar os recursos do programa para que os municípios adquirissem os medicamentos ou deixou de enviar medicamentos aos municípios para dispensação nas farmácias das unidades de saúde;

2. contrapartida SES em desacordo: quando a SES aplicou a contrapartida de forma irregular no aspecto temporal ou quando os valores alocados foram inferiores ao mínimo obrigatório;

3. secretaria municipal de saúde (SMS) não fez contrapartida: casos em que a SMS deixou de alocar os recursos do programa para a aquisição dos medicamentos;

4. contrapartida SMS em desacordo: quando a SMS aplicou a contrapartida de forma irregular no aspecto temporal ou quando os valores alocados foram inferiores ao mínimo obrigatório;

5. falta de comprovação do gasto dos recursos: situação em que o município não apresentou documentos que comprovassem efetivamente que o recurso financeiro foi utilizado para a aquisição de medicamentos;

6. falta de prestação de contas: quando o município deixou de alimentar o Sistema de Acompanhamento do Incentivo Financeiro da Assistência Farmacêutica na Atenção Básica (SIFAB), o qual serve para monitoramento da alocação dos recursos do programa;

7. aquisição de medicamentos em desacordo com a lista pactuada: quando o município adquiriu medicamentos outros que não apenas aqueles constantes na lista obrigatória do programa;

8. aquisição a preço superior ao constante no Banco de Preços em Saúde (BPS): situação em que os preços pagos pelo município para a aquisição de medicamentos foram superiores ao preço médio do medicamento no BPS, um sistema do Ministério da Saúde em que as instituições informam os preços pagos em suas compras;

9. inobservância das normas de aquisição: quando foi constatado que o município deixou de realizar os procedimentos obrigatórios para a realização de compras pelo setor público (licitação) ou quando a licitação foi feita sem observância de todos os requisitos previstos em lei;

10. fraude e desvio de recursos do programa: situação em que foi constatada corrupção e em que os recursos foram aplicados para finalidade diversa do que para a compra de medicamentos;

11. ausência de farmacêutico, seja na coordenação da Assistência Farmacêutica do município, seja para a dispensação de medicamentos sujeitos ao controle especial;

12. controle de estoque ausente ou deficiente: quando foi verificado que o município não faz a programação da aquisição de medicamentos, não possui sistema para controle de estoque (informatizado ou por meio de fichas de prateleira) ou quando possui o sistema mas não o utiliza efetivamente; 
TABELA 1. Critérios adotados pela Controladoria Geral da União para a realização do $12^{\circ}$ ao $22^{\circ}$ sorteio público para fiscalização de municípios, Brasil, 2004 a 2006

\begin{tabular}{|c|c|c|c|}
\hline $\begin{array}{l}\text { Número do } \\
\text { sorteio }\end{array}$ & Critérios & $\begin{array}{l}\text { Total de } \\
\text { municípios } \\
\text { elegíveis }\end{array}$ & $\begin{array}{l}\text { \% de municípios } \\
\text { elegíveis do } \\
\text { total do país }\end{array}$ \\
\hline $12^{\circ}$ & $\begin{array}{l}\text { Municípios por estado }: A C, A P, R O \text { e } R R=1 ; A L=1 ; A M=1 ; B A=5 ; C E=2 ; E S=1 ; G O=3 \\
\qquad M A=2 ; M T=2 ; M S=1 ; M G=8 ; P A=2 ; P B=2 ; P R=4 ; P E=2 ; P I=2 ; R J=2 ; R N=2 ; \\
R S=5 ; S C=3 ; S P=6 ; S E=1 \text { e } T O=2\end{array}$ & & \\
\hline $13^{\circ}$ & Municípios por estado: mesmo que $12^{\circ}$ sorteio & & \\
\hline \multirow[t]{2}{*}{$14^{\circ}$} & $\begin{array}{l}60 \text { municípios com até } 500 \text { mil habitantes, excluídas as capitais e aqueles em período de } \\
\text { carência (sorteados nos } 12 \text { últimos sorteios) }\end{array}$ & 4887 & 87,8 \\
\hline & Municípios por estado: mesmo que $12^{\circ}$ sorteio & & \\
\hline $15^{\circ}$ & $\begin{array}{l}\text { Municípios por estado } a \text { : } A C \text { e } R O=1 ; A P \text { e } R R=1 ; A L=1 ; A M=1 ; B A=5 ; C E=2 ; E S=1 \\
\qquad G O=3 ; M A=2 ; M T=2 ; M S=1 ; M G=7 ; P A=2 ; P B=2 ; P R=4 ; P E=2 ; P I=2 ; R J=2 \\
R N=2 ; R S=5 ; S C=3 ; S P=6 ; S E=1 \text { e } T O=2\end{array}$ & & \\
\hline $16^{\circ}$ & Mesmos critérios que $15^{\circ}$ sorteio & 4835 & 86,9 \\
\hline $17^{\circ}$ & Mesmos critérios que $15^{\circ}$ sorteio & 4823 & 86,7 \\
\hline $18^{\circ}$ & Mesmos critérios que $15^{\circ}$ sorteio & 4819 & 86,6 \\
\hline $19^{\circ}$ & Mesmos critérios que $15^{\circ}$ sorteio & 4813 & 86,5 \\
\hline $20^{\circ}$ & $\begin{array}{l}60 \text { municípios com até } 500 \text { mil habitantes, excluídas as capitais e aqueles em período de } \\
\text { carência (os que foram sorteados nos } 12 \text { últimos sorteios ou tiveram fiscalizações especiais), } \\
\text { sendo sorteadas as áreas de fiscalização para municípios com mais de } 20 \text { mil habitantes em } \\
\text { cinco grupos }^{b}\end{array}$ & 4801 & 86,3 \\
\hline
\end{tabular}

Fonte: Controladoria Geral da União (CGU). Programa de Fiscalização a partir de Sorteios Públicos. Disponível em: http://www.cgu.gov.br/AreaAuditoriaFiscalizacao/ExecucaoProgramas Governo/Sorteios/Municipios/Portarias.asp. Acessado em outubro de 2007.

${ }^{\text {a }} \mathrm{AC}=$ Acre; $\mathrm{AL}=$ Alagoas; $\mathrm{AM}=$ Amazonas; $\mathrm{AP}=$ Amapá; $\mathrm{BA}=$ Bahia; $\mathrm{CE}=$ Ceará; $\mathrm{DF}=$ Distrito Federal; $\mathrm{ES}=$ Espírito Santo; $\mathrm{GO}=\mathrm{Goiás} ; \mathrm{MA}=\mathrm{Maranhão} ; \mathrm{MG}=\mathrm{Minas}$ Gerais; $\mathrm{MS}=\mathrm{Mato}$ Grosso do Sul; MT = Mato Grosso; PA = Pará; PB = Paraíba; PE = Pernambuco; PI = Piaúí PR = Paraná; RJ = Rio de Janeiro; RN = Rio Grande do Norte; RO = Rondônia; RR = Roraima; RS = Rio Grande do Sul; SC = Santa Catarina; SE = Sergipe; SP = São Paulo; TO = Tocantins.

${ }^{\mathrm{b}}$ Grupos de áreas a serem fiscalizadas. Por exemplo, o grupo 2 inclui a área da saúde.

13. falta de medicamentos do programa: situação em que foi constatada a falta de medicamentos do programa em unidades básicas de saúde;

14. condições inadequadas de armazenamento: quando foi constatado que nos almoxarifados, nas centrais de abastecimento farmacêutico ou nas farmácias os medicamentos estavam sujeitos a condições impróprias (expostos à luz solar, em contato direto com pisos e paredes, sujeitos a altas temperaturas ou umidade e fora de refrigeração, quando esta é necessária);

15. medicamentos vencidos: situação na qual foram encontrados medi- camentos com prazo de validade expirado nos almoxarifados, centrais de abastecimento farmacêutico ou nas farmácias.

Os dados coletados foram tabulados para a realização de análise exploratória. 


\section{RESULTADOS}

No período de agosto de 2004 a julho de 2006, a CGU realizou 11 sorteios (do $12^{\circ}$ ao $22^{\circ}$ ), resultando na execução de 660 fiscalizações de municípios (todos os sorteados). Nenhum município foi fiscalizado duas ou mais vezes, portanto, as 660 fiscalizações correspondem a 660 municípios.

Cada fiscalização gerou um relatório, que foi disponibilizado no site do órgão na Internet. Um dos 660 relatórios de fiscalização não estava disponível em arquivo no momento da coleta de dados. Portanto, analisaram-se os 659 relatórios disponíveis. Em 62 municípios, a ação Incentivo Financeiro a Municípios Habilitados à parte Variável do Piso de Atenção Básica (PAB) para a Assistência Farmacêutica Básica não foi fiscalizada e, conseqüentemente, os serviços farmacêuticos também não. Desse modo, a amostra deste estudo é composta de 597 relatórios de fiscalização (597 municípios), o que corresponde ao universo de 10,7\% dos municípios brasileiros. A tabela 2 apresenta o número de municípios fiscalizados por região do país.

A análise das irregularidades encontradas na assistência farmacêutica revelou que $90,3 \%$ dos municípios fiscalizados apresentaram pelo menos uma das categorias de problemas criadas nesta pesquisa para agrupar os relatos feitos pelos fiscais da CGU. A tabela 3 resume os percentuais de municípios fiscalizados com problemas e a tabela 4 apresenta a freqüência de problemas relatados no país e por região. A figura 1 apresenta a freqüência dos problemas encontrados nos municípios em que também foi constatada a falta de medicamentos.

\section{DISCUSSÃO}

Considerando os critérios de sorteio utilizados pela CGU para definir os municípios que são fiscalizados, é possível dizer que a presente amostra é representativa do conjunto de municípios do país. Ainda que tenham sido incluídos somente municípios com até 500 mil habitantes, e excluídas as capi-

TABELA 2. Porcentagem de municípios cujo programa de assistência farmacêutica foi fiscalizado, Brasil, 2004 a 2006

\begin{tabular}{lccc}
\hline \multicolumn{1}{c}{ Região } & $\begin{array}{c}\text { Municípios } \\
\text { fiscalizados }\end{array}$ & $\begin{array}{c}\text { Total de } \\
\text { municípios }^{a}\end{array}$ & $\begin{array}{c}\% \text { municípios } \\
\text { fiscalizados do } \\
\text { total de municípios }\end{array}$ \\
\hline Nordeste & 188 & 1793 & 10,49 \\
Norte & 44 & 449 & 9,80 \\
Sudeste & 159 & 1668 & 9,53 \\
Sul & 123 & 1188 & 10,35 \\
Centro-Oeste & 83 & 466 & 17,81 \\
\multicolumn{1}{c}{ Total } & 597 & 5564 & 10,73 \\
\hline Fonte: & & &
\end{tabular}

Fonte: Controladoria Geral da União.

a Conforme Instituto Brasileiro e Geografia e Estatística (IBGE) (13).

TABELA 3. Municípios cujo programa de assistência farmacêutica foi fiscalizado e apresentou problemas, Brasil, 2004 a 2006

\begin{tabular}{lcccc}
\hline So. municípios & $\begin{array}{c}\text { Municípios } \\
\text { fiscalizados } \\
\text { sorteados }\end{array}$ & $\begin{array}{c}\text { Municípios com } \\
\text { problemas }\end{array}$ & $\begin{array}{c}\text { \% de municípios } \\
\text { com problemas }\end{array}$ \\
\hline 12 & 60 & 60 & 56 & 93,3 \\
13 & 60 & 60 & 55 & 91,7 \\
14 & 60 & 60 & 57 & 95,0 \\
15 & 60 & 36 & 33 & 91,7 \\
16 & 60 & 44 & 37 & 84,1 \\
17 & 60 & 47 & 43 & 91,5 \\
18 & 60 & 51 & 43 & 84,3 \\
19 & 60 & 60 & 54 & 90,0 \\
20 & 60 & 60 & 51 & 85,0 \\
21 & 60 & 59 & 53 & 89,8 \\
22 & 60 & 60 & 57 & 95,0 \\
Total & 660 & 597 & 539 & 90,3 \\
\hline
\end{tabular}

Fonte: Controladoria Geral da União.

tais, dos 5564 municípios brasileiros apenas 35 possuem mais de $500 \mathrm{mil}$ habitantes, e desses, 20 são capitais dos estados e do Distrito Federal (13).

É necessário destacar que os municípios com população superior a $500 \mathrm{mil}$ habitantes possuem uma rede de serviços de saúde, e também de assistência farmacêutica, bastante complexa, tanto quantitativa quanto qualitativamente, constituindo-se em referência para os municípios de pequeno e médio porte. Portanto, a sua não inclusão no programa de fiscalizações da CGU, estudado neste trabalho, implica na desconsideração de municípios importantes do ponto de vista da complexidade da oferta, o que pode gerar dificuldades adicionais de gestão. Assim, outras pesquisas precisam ser realizadas para estudar esse universo. De todo modo, o fato de que as fiscalizações da CGU focam a organização municipal da assistência farmacêutica, analisando o conjunto dos serviços em cada município, torna a abordagem mais macro e, conseqüentemente, a quantidade de serviços terá impacto menor na análise dos resultados, pois a unidade de análise é o município, e não o serviço farmacêutico.

A definição do número de municípios a ser sorteados por estado, guardando proporcionalidade com a quantidade de municípios por estado, assegura que todas as regiões do país 
TABELA 4. Frequêencia de problemas na assistência farmacêutica relatados nos relatórios de fiscalização da Controladoria Geral da União, Brasil, 2004 a 2006

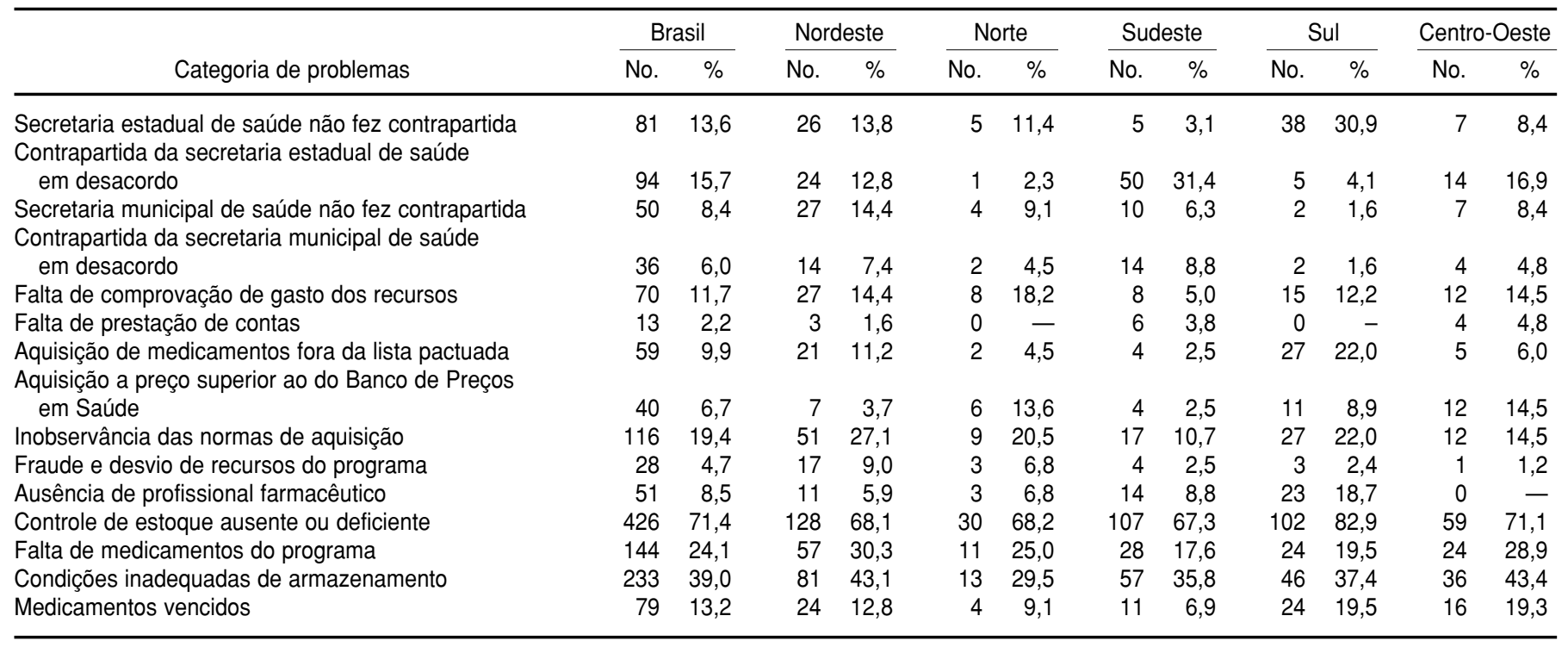

FIGURA 1. Freqüência de problemas apresentados pela Controladoria Geral da União em municípios onde também foi constatada a falta de medicamentos, Brasil, 2004 a $2006^{\mathrm{a}}$

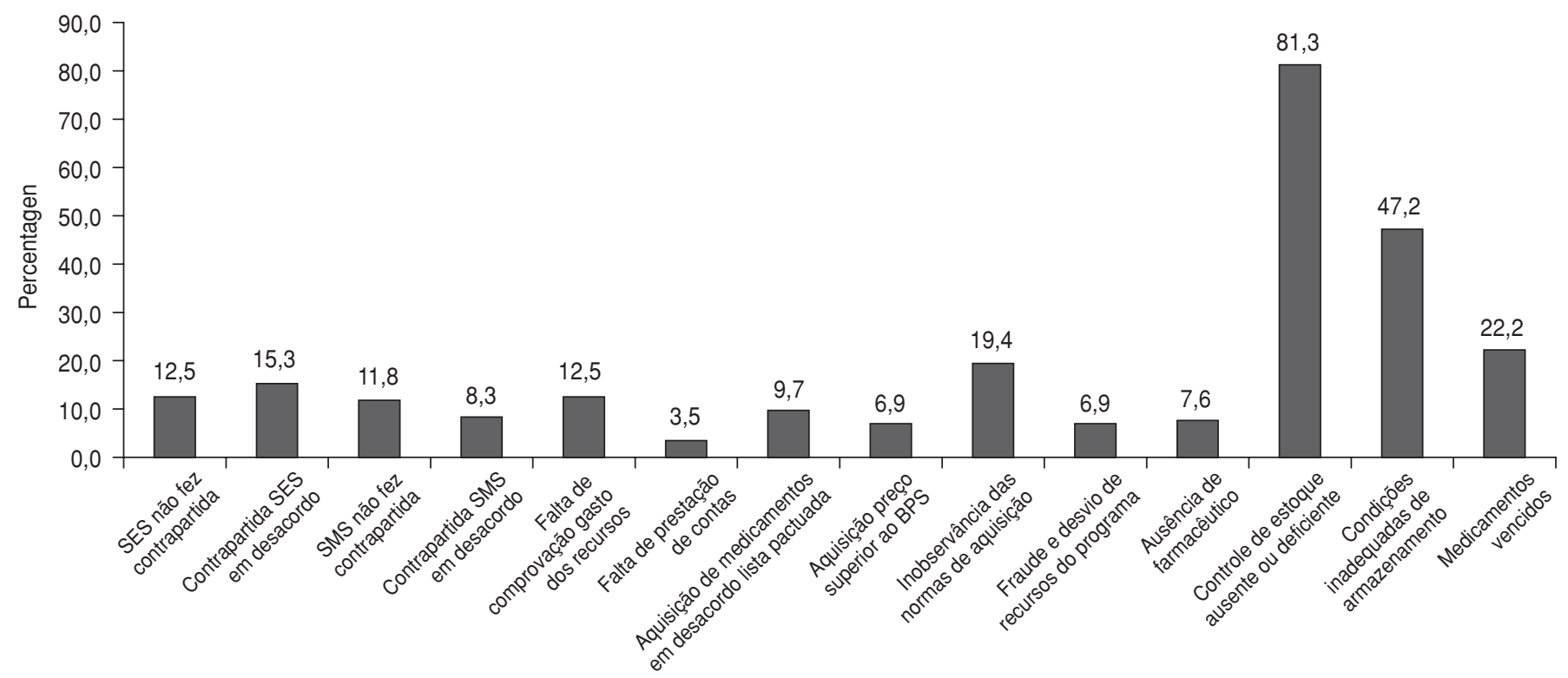

a Total de municípios = 144; SES = secretaria estadual de saúde; SMS = secretaria municipal de saúde .

estejam contempladas. Isso é importante devido à tão conhecida desigualdade de desenvolvimento regional no Brasil. Além disso, a aleatoriedade do sorteio possibilita que, respeitados os critérios mencionados, os municípios do estado tenham a mesma chance de fazer parte da amostra.

O percentual de municípios com problemas dimensiona as dificuldades ainda existentes no SUS para ofertar serviços farmacêuticos com a quali- dade necessária para garantir o acesso e o uso racional de medicamentos. Menos de 10\% deles mostraram-se adequados sob o olhar da auditoria da CGU. A partir da análise das categorias de problemas, fica evidente que 
tarefas básicas de gestão não estão sendo realizadas a contento (tabela 4 ). Os fatos de que $71 \%$ dos municípios apresentaram "controle de estoque ausente ou deficiente" e de que em 39\% deles foram observadas "condições inadequadas de armazenamento" refletem falhas graves de gestão.

A ausência ou deficiência de programação e controle de estoque indica que a aquisição de medicamentos é realizada sem a utilização de parâmetros concretos para a mensuração da oferta conforme a demanda. Assim, os medicamentos podem ser adquiridos ou em quantidade muito superior ao necessário, ocasionando perdas e desperdícios de recursos públicos pela necessidade de descartar produtos com prazo de validade expirado, ou em quantidade muito inferior, prejudicando o acesso da população e, conseqüentemente, a efetividade do cuidado à saúde.

Nesse sentido, a verificação, neste estudo, da falta de medicamentos selecionados para a lista do programa em $24 \%$ dos municípios, com prejuízo ao acesso e à garantia de integralidade da assistência à saúde, um dos princípios do SUS, revela a dimensão do problema. Essa situação pode constituir um dos motivos pelos quais os cidadãos acabam procurando assegurar o seu direito de acesso a medicamentos pela via judicial. Um estudo recente revelou que, no caso do Município de São Paulo, 62\% dos medicamentos demandados por meio de ações judiciais constavam na lista de medicamentos essenciais (14).

A outra conseqüência da falta de programação e controle de estoque consiste na ineficiência do uso dos recursos públicos. Em 13\% dos municípios foram encontrados medicamentos vencidos (prazo de validade expirado). As perdas de medicamentos podem ser agravadas pelas condições inadequadas de armazenamento, situação esta observada em 39\% dos municípios. O fato sinaliza a falta de investimento na estrutura de centrais de abastecimento farmacêutico e de locais apropriados para o armazenamento nas farmácias das unidades de saúde e, ainda, em recursos humanos.
Outro fator que pode causar ineficiências é a aquisição de medicamentos que não constam na lista do programa, situação que foi constatada em quase $10 \%$ dos municípios. Considerando que a seleção desses medicamentos é feita com base em critérios de essencialidade, observando a Relação Nacional de Medicamentos Essenciais (RENAME), a aquisição de itens diferentes dos selecionados pode resultar na oferta de medicamentos com uma relação custo-efetividade desfavorável.

Embora alguns municípios desconsiderem a RENAME quando selecionam medicamentos para oferta nos serviços de saúde, o conceito de medicamento essencial e a sua aplicabilidade continuam tão atuais e úteis quanto há 30 anos (15). Estima-se que $70 \%$ dos produtos farmacêuticos no mercado mundial estejam duplicados ou não sejam essenciais, e que muitos não passem de pequenas variações moleculares de um protótipo, sem vantagens terapêuticas sobre fármacos que já estão disponíveis (16). Instituições como a Organização Mundial da Saúde (OMS) acreditam que, com uma gama enorme de medicamentos sendo comercializados, é quase impossível aos prescritores manterem-se atualizados e compararem as alternativas existentes, e que, para os sistemas de saúde, a extensa variedade de produtos farmacêuticos pode gerar prescrições inconsistentes e contribuir para o aumento dos custos, já que se perde a economia de escala (16).

Ainda em relação ao uso dos recursos, os relatórios de fiscalização da CGU mostram que 19\% dos municípios não observaram as normas vigentes para a realização de compras públicas. Embora essas normas tornem o processo de aquisição de medicamentos ou de qualquer outro bem e serviço mais moroso, o seu objetivo precípuo é o de conferir economia e transparência aos atos administrativos relacionados. $\mathrm{O}$ percentual encontrado indica que podem estar ocorrendo perdas devido à inobservância das normas, à ausência de qualificação e ao treinamento do pessoal responsável por sua execução. Questões mais graves, como fraudes e desvios de recursos, foram identificadas em aproximadamente $5 \%$ dos municípios fiscalizados.

Em relação aos recursos humanos, em cerca de 9\% dos municípios verificou-se a ausência de farmacêutico na coordenação ou dispensação de medicamentos sujeitos ao controle especial. Embora não haja dados do SUS sobre a relação de farmacêuticos por município ou serviços de saúde, o número parece subestimado diante da experiência de alguns municípios, relatada por profissionais que atuam tanto em secretarias municipais quanto estaduais de saúde. Outros levantamentos precisam ser realizados para averiguar essa questão. De todo modo, o fato de que há municípios sem farmacêuticos indica uma fragilidade. Entretanto, a simples presença do profissional não garante a qualidade do serviço. Em alguns casos, por exemplo, ele acaba assumindo apenas um papel administrativo, distanciando-se da atenção ao paciente (17).

Nesse sentido, a OMS assevera que a boa prática da farmácia deve focar quatro grupos de atividades, cujo desenvolvimento deve se dar com a atuação de farmacêuticos: a) as atividades associadas à promoção da saúde; b) as relacionadas à disponibilidade de medicamentos; c) as relacionadas ao autocuidado; e d) as relacionadas com as prescrições e o uso racional de medicamentos (18).

Nos últimos anos, tem sido demonstrada e discutida a importância da presença de farmacêuticos nas equipes de saúde $(19,20)$. Por exemplo, uma revisão sistemática sobre o impacto da análise e do monitoramento de prescrições por farmacêuticos em serviços ambulatoriais concluiu que esse procedimento pode melhorar as condições clínicas dos pacientes e reduzir o custo dos medicamentos prescritos (21). Em outro estudo, fez-se uma análise de custo-efetividade da orientação farmacêutica para pacientes idosos em uso ambulatorial de medicamentos e constatou-se que a inclusão de um protocolo para essa orientação reduziu os custos das prescrições dos pacientes, embora não tenha ocorrido impacto significativo no uso dos serviços de saúde (22). 
Retornando à questão dos recursos financeiros, em relação àqueles que estados e municípios devem alocar para a aquisição de medicamentos, este estudo revela que, em $14 \%$ dos municípios, as SES deixaram de efetuar a sua contrapartida. Desses $14 \%, 12 \%$ das SMS também não aplicaram os recursos, e em $4 \%$ a contrapartida municipal foi feita em desacordo com o estabelecido. Além disso, em 16\% dos municípios, as SES fizeram a sua contrapartida de forma irregular. Em resumo, em 29\% dos 597 municípios fiscalizados houve problemas de efetivação da contrapartida estadual e em $14 \%$ da contrapartida municipal. Essa situação é altamente comprometedora dos objetivos do programa, que é ampliar o acesso da população aos medicamentos essenciais. A ausência ou irregularidade de alocação dos recursos faz com que estejam disponíveis à população somente aqueles alocados pelo Ministério da Saúde, o que corresponde a $50 \%$ do valor mínimo obrigatório per capita por ano, insuficientes para financiar a aquisição dos medicamentos selecionados (2).

Quando se analisam os dados regionais constantes na tabela 4 , observa-se que na Região Sul quase 31\% dos municípios não receberam os recursos do programa referentes à contrapartida estadual. O não repasse desses recursos provavelmente foi responsável pela realização de maior esforço dos municípios em alocar os valores devidos a título de contrapartida municipal, pois é dessa Região o menor percentual de municípios que não alocaram os recursos (1,6\%). É importante destacar que, quando comparada às demais regiões, é também da Região Sul o maior percentual de municípios que: a) adquiriram medicamentos em desacordo com a lista pactuada $(22 \%)$, b) não tinham farmacêutico $(18,7 \%)$, c) não faziam controle de estoque ou tinham controle deficiente $(82,9 \%)$ e d) tinham medicamentos vencidos (19,5\%). Esses dados sugerem que a menor participação da SES no financiamento e gestão do programa pode afetar o desempenho dos municípios no gerenciamento dos serviços farmacêuticos. Entretanto, mais estudos são necessários para analisar as relações de causalidade desses problemas e suas inter-relações.

Ainda na tabela 4, nota-se que 3\% dos municípios da Região Sudeste não receberam os recursos da contrapartida estadual; entretanto, $31 \%$ de todos eles receberam esse recurso em desacordo com o pactuado. Os maiores percentuais de fraudes e desvios foram relatados em municípios das regiões Nordeste ( $9 \%$ ) e Norte $(6,8 \%)$, e de falta de medicamentos também, sendo que o Nordeste ficou em primeiro lugar $(30,3 \%)$, o Centro-Oeste em segundo $(28,9 \%)$ e o Norte em terceiro (25\%).

$O$ resultado desse quadro é que certamente o sistema de saúde não consegue cumprir o requisito fundamental para o uso racional de medicamentos, que é o da oportunidade de oferta do tratamento. As conseqüências disso acabam se somando, tais como agravamento do quadro de saúde do paciente e necessidade de tratamentos mais complexos ou internação, aumento dos gastos e ineficiência na alocação de recursos.

Sobre a falta de medicamentos, nota-se na figura 1 que os problemas mais freqüentes nos municípios nos quais foi constatada a indisponibilidade dos produtos farmacêuticos selecionados para o programa foram: controle de estoque ausente ou deficiente (81\%), condições inadequadas de armazenamento (47\%), contrapartida estadual ausente ou em desacordo $(28 \%)$, medicamentos vencidos $(22 \%)$, contrapartida municipal ausente ou em desacordo (20\%) e inobservância das normas de aquisição (19\%). Esses achados sugerem que o estabelecimento de programação e o controle eficiente de estoques são determinantes para evitar a falta de medicamentos, mais do que a alocação adequada dos recursos do programa. Outro dado importante é o percentual significativo de medicamentos vencidos quando há falta de medicamentos, indicando mais uma vez a ineficiência dos controles de estoque.

Se, de um lado, o acesso a medicamentos essenciais é fundamental para a melhora ou a manutenção do estado de saúde da população, por outro, o uso inadequado de medicamentos constitui-se em risco de problemas sérios, com redução da qualidade de vida e aumento de custos para o sistema de saúde. Daí a necessidade de se promover o uso racional de medicamentos, de forma a garantir que os pacientes recebam o medicamento correto nas doses adequadas, pelo tempo necessário e ao menor custo possível, tanto para o próprio paciente quanto para a comunidade (23).

Para que a situação de uso racional de medicamentos se torne realidade, é preciso que os serviços farmacêuticos cumpram o seu papel. Não é possível falar em uso racional se há falta de medicamentos essenciais; se são disponibilizados medicamentos com menor eficácia, segurança, efetividade e razão de custo-efetividade incremental desfavorável em relação a outros disponíveis no mercado; se não há garantia de preservação da qualidade dos medicamentos quando de seu armazenamento ou se os pacientes os recebem sem qualquer orientação sobre o uso.

Esse é justamente o quadro que este estudo revela. Embora a pesquisa apresente limitações decorrentes do fato de que os auditores da CGU não são profissionais que conhecem profundamente o tema e de que são grupos diferentes de auditores que realizam o trabalho, o que aumenta o número de olhares sobre as situações constatadas e assim a sua variabilidade, essas limitações não são suficientes para invalidar os resultados ora apresentados. $\mathrm{O}$ estudo mostra-se útil para diagnosticar o problema concreto de qualidade dos serviços farmacêuticos no SUS e seus achados vão ao encontro de outra pesquisa realizada com o mesmo objetivo (11).

Está claro que o propósito de reorientação da assistência farmacêutica, previsto na PNM, e o de qualificação dos serviços farmacêuticos, explicitado na PNAF, estão muito aquém de sua concretização, como mostra este estudo, que evidencia os detalhes dessa agenda inconclusa. É preciso avançar muito e superar o modelo vigente de assistência farmacêutica no SUS, reduzido na maioria dos municípios a aquisição e distribuição de medicamentos. 
Ainda que alguns avanços tenham sido alcançados (24), faz-se necessário fortalecer a gestão, até mesmo porque o modelo atual mostra-se ultrapassado e ineficiente.

De acordo com Marin et al. (5), a adoção de um enfoque sistêmico para a organização da assistência farmacêutica é uma estratégia de superação da lógica fragmentada da área, ou seja, apenas a valorização de ações de aquisição, armazenamento e distribuição. Apresentada de forma sistêmica, a assistência farmacêutica integra os componentes de natureza técnica, científica e operativa, conforme a complexidade dos serviços, necessidades e finalidades.

Para tanto, os entes que integram o SUS - União, estados e municípios - precisam perceber-se a si mesmos como unidades de um mesmo sistema e cooperar uns com os outros para que o esforço de reorientação da assistência farmacêutica e de qualificação dos serviços farmacêuticos se traduza em garantia de acesso a medicamentos essenciais, com uso racional.

Essa cooperação entre os gestores do SUS também precisa se firmar com o apoio das universidades, organismos de cooperação internacional, conselhos de classe e sociedades civis afins, conformando uma rede alinhada conceitualmente e empenhada para o alcance dos objetivos e metas propostas, visando à transformação da realidade. Somente o esforço conjunto possibilitará a superação das dificuldades ora encontradas, para que o SUS efetivamente ofereça à população serviços farmacêuticos de alta qualidade.

Por fim, é preciso enfatizar que a assistência farmacêutica precisa retornar às agendas dos governos, especialmente do Governo Federal, devido à sua capacidade de mobilização das instâncias subnacionais, de financiamento e de formulação de prioridades e diretrizes. Os governos precisam assumir o compromisso de implementar as propostas explicitadas nas políticas vigentes e nos relatórios das conferências de saúde e das conferências de assistência farmacêutica, pois não é possível falar em efetividade da atenção à saúde sem que a assistência farmacêutica cumpra o seu papel com qualidade.

\section{REFERÊNCIAS}

1. Brasil, Constituição da República Federativa do Brasil. Texto consolidado até a Emenda Constitucional $\mathrm{n}^{\circ} 55$ de 20 de setembro de 2007. Brasília: Senado Federal; 2007. Disponível em: http://www.senado.gov.br/sf/ legislacao/const/. Acessado em outubro de 2007.

2. Rua MG. Análise de políticas públicas: conceitos básicos. Em: Rua MG, Carvalho MIV. O estudo da política: estudos selecionados. Brasília: Paralelo 15; 1998. Pp. 231-61.

3. Brasil, Ministério da Saúde. Política Nacional de Medicamentos. Brasília: Ministério da Saúde; 2001. Disponível em: http://bvsms. saude.gov.br/bvs/publicacoes/politica medicamentos.pdf. Acessado em outubro de 2007.

4. Brasil, Ministério da Saúde. Incentivo à assistência farmacêutica básica: o que é e como funciona. Brasília: Ministério da Saúde; 2001. Disponível em: http://bvsms.saude.gov.br/ bvs/publicacoes/incentivo_assit_farm.pdf. Acessado em outubro de 2007.

5. Marin N, Luiza VL, Osório-de-Castro CGS, Machado-dos-Santos S, orgs. Assistência farmacêutica para gerentes municipais. Rio de Janeiro: OPAS/OMS; 2003. Disponível em: http://www.opas.org.br/medicamentos / site/UploadArq/0080.pdf. Acessado em outubro de 2007.

6. Brasil, Ministério da Saúde. Assistência farmacêutica na atenção básica. Instruções técnicas para sua organização. Brasília: Ministério da Saúde; 2002. Disponível em: http:/ /bvsms. saude.gov.br/bvs/publicacoes/cd03_15.pdf. Acessado em outubro de 2007.

7. Brasil, Conselho Nacional de Saúde. Resolução ${ }^{\circ} 338$ de 6 de maio de 2004. Aprova a política nacional de assistência farmacêutica.
Disponível em: http://portal.saude.gov.br/ portal/arquivos/pdf/resol_cns338.pdf. Acessado em outubro de 2007.

8. Brasil, Ministério da Saúde. Planejar é preciso: uma proposta de método para aplicação à assistência farmacêutica. Brasília: Ministério da Saúde; 2006. Disponível em: http://bvsms. saude.gov.br/bvs/publicacoes/06_1143_M. pdf. Acessado em outubro de 2007.

9. Brasil, Ministério da Saúde. Aquisição de medicamentos para assistência farmacêutica no SUS. Brasília: Ministério da Saúde; 2006. Disponível em: http://portal.saude. gov.br/portal/arquivos/pdf/aquisicao_ de_medicamentos_para_a_assistencia_ farmaceutica_no_sus.pdf. Acessado em outubro de 2007.

10. Brasil, Ministério da Saúde. Assistência farmacêutica na atenção básica. Instruções técnicas para sua organização. $2^{\mathrm{a}}$ ed. Brasília: Ministério da Saúde; 2006. Disponível em: http:// portal.saude.gov.br/portal/arquivos/pdf/ assistencia_farmaceutica_na_atencao_ basica.pdf. Acessado em outubro de 2007.

11. Organização Pan-Americana da Saúde, Ministério da Saúde. Avaliação da assistência farmacêutica no Brasil: estrutura, processo e resultados. Brasília: Organização Pan-Americana da Saúde; 2005. Disponível em: http://www. opas.org.br/medicamentos/site/Upload Arq/HSE_ASF_REM_1205.pdf. Acessado em outubro de 2007.

12. Banco Mundial. Governança no Sistema Único de Saúde (SUS) do Brasil. Melhorando a Qualidade do Gasto Público e Gestão de Recursos. Washington: Banco Mundial; 2007. Disponível em: http://www-wds. worldbank.org/external/default/WDS ContentServer/WDSP /IB / 2007/07/18/
000090341_20070718142655/Rendered/PDF/ 3660110portuguese.pdf. Acessado em outubro de 2007.

13. Instituto Brasileiro de Geografia e Estatística (IBGE). Contagem da população 2007. Rio de Janeiro: IBGE; 2007. Disponível em: http://www.ibge.gov.br/home/estatistica/ populacao/contagem2007/popmunic2007 layoutTCU14112007.xls. Acessado em maio de 2008.

14. Vieira FS, Zucchi P. Distorções causadas pelas ações judiciais à política de medicamentos no Brasil. Rev Saude Publica. 2007;41(2):214-22.

15. Organización Mundial de la Salud. Perspectivas políticas sobre medicamentos de la OMS 4. Selección de medicamentos esenciales. Genebra: OMS; 2002. Disponível em: http:// whqlibdoc.who.int/hq/2002/WHO_EDM_ 2002.2_spa.pdf. Acessado em outubro de 2007.

16. World Health Organization. Public-private roles in the pharmaceutical sector: implications for equitable access and rational use. Health Economics and Drugs DAP Series No. 5, Action Programme on Essential Drugs. Genebra: WHO; 1997.

17. Neves JOS, Silver LD. Evaluation of pharmaceutical assistance in public primary care in Brasília, Brazil. Rev Saude Publica. 2005;39(2): 223-30.

18. Organización Mundial de la Salud. Buenas prácticas de farmacia: normas de calidad de servicios farmacéuticos. Disponível em: http://www.opas.org.br/medicamentos/ site/UploadArq/ops-hss-hse-95-01.pdf. Acessado em outubro de 2007.

19. Vieira FS. Possibilidades de contribuição do farmacêutico para a promoção da saúde. Cienc Saude Coletiva. 2007;12(1):213-20. 
20. Timbs O. Leading role for pharmacists to reduce errors and improve patient safety. Pharm J. 2002;268(7190):392.

21. Tully MP, Seston EM. Impact of pharmacists providing a prescription review and monitoring service in ambulatory care or community practice. Ann Pharmacother. 2000;34(11): 1320-31.

22. Cowper PA, Weinberger M, Hanlon JT, Landsman PB, Samsa GP, Uttech KM, et al. The cost-effectiveness of a clinical pharmacist intervention among elderly outpatients. Pharmacotherapy. 1998;18(2):327-32.

23. Organización Mundial de la Salud. Promoción del uso racional de medicamentos: componentes centrales. Genebra: OMS; 2002. Disponível em: http://www.who.int/ medicinedocs/collect/edmweb/pdf/s4874s/ s4874s.pdf. Acessado em outubro de 2007.

24. Brasil, Ministério da Saúde, Universidade Federal de Minas Gerais. Organização da assistência farmacêutica nos municípios brasi- leiros: disponibilidade e utilização de medicamentos no SUS: pesquisa telefônica. Brasília: Ministério da Saúde; 2002. (Série B. Textos Básicos de Saúde.)

Manuscrito recebido em 26 de outubro de 2007. Aceito em versão revisada em 6 de fevereiro de 2008.

ABSTRACT Objective. To identify items on the agenda of Brazil's Unified Health System that are related to the quality of pharmaceutical services and have not yet been implemented. Methods. Data were collected from the inspection reports issued by the federal gov-

Providing quality pharmaceutical services in Brazil: Items pending on the Unified Health System's agenda ernment's controllership office. Inspection reports completed from August 2004-July 2006, available on the Internet, were reviewed. The issues identified were classified into 15 categories.

Results. Of the 660 inspections that were carried out, 659 were analyzed (one was unavailable). In 62 cities pharmaceutical assistance services had not been audited, therefore, a the final sample of 597 cities was analyzed (10.7\% of Brazil's municipalities). Of these, $90.3 \%$ had issues associated with the management of resources or services. In $71 \%$ of the municipalities, the reports indicated lack of or deficient inventory control, and in 39\%, inadequate storage conditions. A shortage of drugs was observed in $24 \%$ of the municipalities.

Conclusions. The goals of Brazil's national drug and pharmaceutical assistance policies are far from being met. Quality pharmaceutical services must again be included on the agenda at all three government levels since it is impossible to have effective health care without quality pharmaceutical services.

Key words Pharmaceutical services, process assessment, National Policy of Pharmaceutical Assistance, National Drug Policy, Single Health System, Brazil. 